\title{
Los trabajos prácticos en la enseñanza de la Biología evolutiva y la Biología funcional: paralelos epistemológicos y didácticos.
}

\section{The practical work in the teaching of evolutionary biology and functional biology: parallel epistemological and didactic}

Por: Germán Alberto Cháves Mejía. ${ }^{1}$

\begin{tabular}{|l|}
\hline Recibido: $19-02-2010$ \\
\hline Aceptado: $27-05-2010$
\end{tabular}

"Soy de las que piensan que la Ciencia tiene una gran belleza. Un sabio en su laboratorio no es solamente un teórico, es también un niño colocado ante los fenómenos naturales que le impresionan como un cuento de hadas".

\section{Marie Curie ${ }^{2}$}

\section{Resumen}

A través de un paralelo epistemológico y didáctico entre las metodologías propias de la biología funcional y evolutiva, evidenciar algunos aspectos en los que coinciden y difieren éstos dos enfoques, en relación con la enseñanza de la Biología en las aulas con el fin de hacer una reflexión que pueda mejorar los procesos de enseñanzaaprendizaje de la Biología.

\section{Palabras claves}

Biología evolutiva, Biología funcional, trabajos prácticos, experimento, observación.

\begin{abstract}
Through an epistemological and didactic parallel between evolutionary and functional Biology methodologies, it is aimed to prove some aspects in which these approaches agree and disagree with, in relation to Biology teaching in the classroom so that a reflection can improve Biology teaching and learning processes.
\end{abstract}

Keywords: Evolutionary biology, functional biology, practical works, experiments, , observation.

\footnotetext{
${ }^{1}$ Biólogo, Universidad Nacional, Bogotá. Especialista en la Enseñanza de la Biología, Universidad Pedagógica Nacional, Bogotá. E-mail gchavesm@ecci.edu.co

${ }^{2}$ Frase inédita pronunciada por Marie Curie en el marco de su catedra de Física en la Universidad de Sorbona (Paris), 1906.
} 


\section{Introducción}

A pesar de que la Biología y su didáctica corresponden a campos de estudio ampliamente diferenciados, cuando el estudio de lo vivo se ve inmerso en el campo de la enseñanza surgen relaciones entre estos dos campos desde lo metodológico como desde lo epistemológico, en consecuencia, se pueden distinguir dos metodologías más o menos diferenciadas para acercarse a la enseñanza de la Biología, una que indaga principalmente sobre el por qué del fenómeno como tal- la Biología evolutiva-, y otra, -la Biología funcional-, que indaga más sobre el cómo de los procesos acaecidos en los organismos vivientes.

En este orden de ideas, el objeto del presente ensayo es evidenciar cómo en la enseñanza de la Biología al utilizar las estrategias didácticas conocidas como trabajos prácticos, éstas pueden variar dependiendo de si el enfoque de enseñanza apunta más hacia una Biología evolutiva o a una Biología funcional. Es importante aclarar que al utilizar trabajos prácticos para la enseñanza de cualquiera de los dos enfoques de la Biología (evolutivo o funcional) podrían también existir puntos en común en la didáctica y epistemología de los mismos, reconocer lo anterior en los docentes podría favorecer los procesos de enseñanza-aprendizaje en la escuela.

Diferencias y similitudes epistemológicas entre el enfoque evolutivo y funcional de la Biología.

El enfoque en Biología evolutiva. Debido a que prácticamente hasta la segunda mitad del siglo XIX, los estudios sobre seres vivos fueron realizados por naturalistas como Linneo, Paley, Buffon, Lamarck, Darwin, entre otros, todos ellos basados en la observación y comparación como fuente de conocimiento, no sería de extrañarse que en los estudios evolutivos el método observacional-comparativo sea preponderante. Lo anterior, debido a que muchos de los naturalistas mencionados trataron de acercarse a dar una explicación de algunos aspectos de lo vivo como son: la clasificación (taxonomía), variabilidad, transformación, que hoy en día, podemos asociar con la teoría evolutiva. Aunque algunos planteamientos de ellos han sido revaluadas (Lamarck y anteriores), otras han sido aceptadas por la comunidad científica (Darwin y la evolución por selección natural), es indudable que los métodos propios de los naturalistas han permeado la metodología de estudio de la Biología evolutiva.

Teniendo en cuenta que la Biología evolutiva trata de los hechos, las regularidades y los procesos en la evolución de los seres vivos y que una de las características más significativas de esa evolución es la generación de diversidad biológica a diferentes niveles, desde los subespecíficos hasta los grandes grupos taxonómicos, la explicación a 
estos fenómenos es sin duda como lo expresa Mayr (2006) buscar respuestas desde el "¿por qué?" y las causas remotas. Este tipo de explicaciones en muchas ocasiones hay que hacerlas desde la observación, comparación, narrativa histórica, e inferencias. Mayr, ejemplifica esto muy bien al decirnos: "no es posible hacer experimentos acerca de la extinción de los dinosaurios o el origen del hombre. Con el experimento no disponible para la investigación en materia de Biología Histórica, se ha introducido un notable y nuevo método heurístico, el de las narrativas históricas" (Mayr, 2006, p.50).

Por otra parte, al estar la Biología evolutiva ligada el estudio de las causas remotas, éstas tienen la peculiaridad de no sólo que "han actuado durante largos períodos, y más concretamente en el pasado evolutivo de la especie" (Mayr, 1995, p. 137); sino que también presentan la singularidad de que sus efectos se registran a nivel poblacional y no individual (Mayr, 1995).

Es importante anotar, que en algunos casos, como en los estudios de genética de poblaciones, estudios relacionados a la Biología evolutiva, es posible la aplicación de técnicas experimentales diferentes a las mencionadas por Mayr, aunque con ciertas limitantes (Caponi, 2003).

En cuanto a la observación en Biología, la podemos definir como una percepción intencionada e ilustrada, consiste en el examen atento de los hechos sobre los que se realiza una investigación determinada. De este modo, se puede analizar un objeto o fenómeno para encontrar sus particularidades o rasgos específicos. En Biología se emplea la observación para identificar las características de los seres vivos y va relacionada con la comparación en Biología que consiste en el procedimiento que se utiliza para encontrar diferencias y semejanzas entre los objetos o procesos que se estudian.

Considerando lo anterior, se puede retomar el pensamiento de Caponi que nos dice "la observación científica no solo es metódica y ordenada, sino que también ya sabemos que la misma exige una activa búsqueda de lo que debemos y queremos observar...la observación es una realización práctica...algo que como Popper gustaba decir es algo que se hace y no algo que se tiene" (Caponi, 2003. p. 70).

No sobra resaltar que este tipo de observaciones científicas utilizadas en Biología son de carácter hipotético deductivo y de ninguna manera en concordancia con el pensamiento inductivo de Bacon, ya que él argumentaba que el observador debía llegar al fenómeno sin estar contaminado de conjeturas al respecto, y de esta manera obtener conocimiento del objeto observado, lo cual es una posición insostenible y revaluada por la comunidad científica, en cambio de esto sería bueno retomar una frase de Darwin, en una de sus 
conferencias realizadas en Inglaterra después de su viaje en el Beagle al argumentar "sin especulación no hay ninguna nueva observación"3.

En cuanto a la hermana siamés de la observación: la comparación, podemos retomar la argumentación de Díaz que nos dice al respecto:

"La comparación entre especies (o taxones de orden superior) es una de las herramientas metodológicas más ampliamente utilizadas en todos los ámbitos de la Biología evolutiva, incluyendo aspectos tan variados como el estudio de los ciclos vitales ("life histories"), la abundancia y distribución de los organismos, la ecomorfología, la ecofisiología o la ecología de la conducta. En particular, las comparaciones interespecíficas permiten comprender cómo la herencia ancestral (inercia filogenética) y la selección natural (adaptación), es decir, los dos grandes temas de la biología evolutiva desde Darwin en adelante, han ido moldeando los rasgos que observamos en las especies actuales. Además, los estudios comparativos revelan con frecuencia regularidades sorprendentes" (Díaz, 2002 p.1)

Con estas dos poderosas herramientas, la observación y la comparación se puede indagar las causas remotas, evolutivas, para explicar por qué los organismos son como son, el origen y la historia de los programas genéticos etc. (Mayr 1995).

El enfoque en Biología funcional. En la segunda mitad del siglo XIX, se empiezan a hacer necesario estudiar el funcionamiento mismo de los cuerpos vivos y de sus componentes (Puentes, 2008), a este respecto el fisiólogo Bernard argumenta que solo a través de la experimentación se pueden descubrir las funciones biológicas (Canguilhem, 1976), tratando de dar un orden a lo intraorgánico como son las estructuras, funciones, y actividades que hacen que un organismo viviente se constituya como tal (Caponi, 2003).

En este orden de ideas, la experimentación es una metodología característica más no única de la Biología funcional, aclarando que este tipo de experimentación, en muchas casos no cumple con la concepción de experimento (en sentido estricto), como en la física o la química, en la que se pueden aislar o manipular variables, lo cual es muy difícil de realizar en la experimentación biológica debido al carácter único del fenómeno estudiado por la Biología (fenómeno de lo vivo), que posee entre muchas características las siguientes: que las poblaciones están conformadas por organismos únicos, existen interacciones entre los organismos y su entorno, e incluso un organismo no es el mismo a lo largo del tiempo y mucho menos cuando sobre un organismo se ha realizado algún procedimiento, como una ablación por ejemplo.

\footnotetext{
${ }^{3}$ Es una de sus tantas frases inéditas, con la cual Darwin se dio cuenta de que él no seguía los enfoques inductivistas baconianos que un principio defendió.
} 
Este tipo de experimentación en Biología funcional se acercaría a la comprensión de las causas próximas y estaría en concordancia con el método analítico (no reduccionista), en el que se pretendería fragmentar un sistema complejo hasta llegar a un nivel en que el estudio de esas partes sea productivo, enfoque histórico que da explicación de cómo estudios en anatomía gruesa llevaron a estudios en microscopia o cómo la fisiología de los órganos llevo a la fisiología celular, Mayr (2006).

Así tenemos, que al ser las causas próximas el objetivo (target) principal de la Biología funcional, ella tratará de explicar el funcionamiento de un organismo y sus fragmentos, en un momento dado o en su desarrollo. Sus objetivos variarán desde la morfología funcional hasta la bioquímica y podrán lograr desde la decodificación de programas genéticos hasta programas somáticos (Mayr 1995).

\section{Coincidencias metodológicas entre la Biología evolutiva y la Biología funcional. En este} apartado se evidenciarán algunos puntos que tienen en común las metodologías utilizadas en los dos enfoques de los estudios biológicos.

¿Es posible realizar experimentaciones en estudios evolutivos? Al remontarse a los planteamientos de Caponi (2003) en los que nos habla sobre los experimentos en genética de poblaciones, la respuesta a la pregunta sería un contundente "sí". No cabría duda que los estudios en genética de poblaciones son una experimentación, no en el sentido estricto de experimento usado por la química y la física, con aislamiento y manipulación de variables, pero sí sería una experimentación en el sentido de ser un procedimiento metódico, con un diseño experimental que permite corroborar unas hipótesis previamente planteadas y con alguna posibilidad de poder lograr unas generalizaciones sobre los resultados obtenidos, aunque obviamente por el carácter único del objeto de estudio biológico mencionado anteriormente, estas generalizaciones tendrían sus limitantes. En este sentido se puede inferir que en algunos casos existen coincidencias entre la metodología característica de la Biología funcional y la de la Biología evolutiva.

Ahora bien, ¿qué sucedía antes de los estudios en genética de poblaciones? ¿Existía experimentación en las investigaciones evolutivas? Parece ser que el propio Darwin realizaba algunos procedimientos experimentales diferentes a sus clásicos métodos observacionales y comparativos, evidencia de esto son entre otros sus trabajos sobre orquídeas ${ }^{4}$ en los que hacía escrupulosas y metódicas disecciones de los órganos sexuales de estas plantas o en sus famosas experimentaciones de cruces en especies domésticas de

\footnotetext{
"Se recomienda revisar la obra de Darwin "La Fecundación de las Orquídeas".
} 
animales y plantas realizadas y documentadas en Variations of Animals and Plants ${ }^{5}$ con un enfoque a resolver preguntas del “ipor qué?" o causas remotas evolutivas.

Es decir, que en concordancia con el párrafo anterior, en Biología evolutiva se pueden utilizar algunos procedimientos experimentales que puedan servir para dilucidar las problemáticas propias de este tipo de enfoque de investigación. La clave para el investigador sería saber hacer la conexión entre las experimentaciones y el problema que se intenta indagar, lo anterior se podría ejemplificar de la siguiente manera: si el investigador hace una disección de una flor y sólo se queda en la morfología y fisiología de las estructuras no estaría haciendo Biología evolutiva, por el contrario si al hacer una disección de este tipo intenta inferir relaciones filogenéticas, a partir de lo morfofisiológico con otras especies, estaría indagando sobre preguntas del “¿por qué?” y causas remotas, características de los estudios evolutivos.

¿Qué sucede con la biología funcional? ¿Utiliza metodologías como observación y comparación? La biología funcional se ajusta bien al estudio de muchos aspectos infraorganísmicos, intentando asintóticamente ${ }^{6}$, lograr la manipulación de organismos en condiciones de laboratorio donde pueden repetirse a voluntad y relativa velocidad los procedimientos en estudio, es decir la Biología funcional en muchos casos tiende a parecerse a los métodos usados en Física y Química, sin embargo si todas estas experimentaciones no fueran observadas por ojos inquietos, heurísticos y curiosos (observación metódica) que busquen relaciones, diferencias similitudes (método comparativo), gran parte de la parafernalia investigativa de la Biología funcional podría ser estéril.

\section{Consideraciones didácticas en trabajos prácticos.}

A continuación se revisarán algunas concepciones con respecto al término trabajo práctico, con el fin de contextualizar las consideraciones de este apartado.

“Según Luís del Carmen (2000), el Trabajo Práctico puede ser considerado, como aquellas actividades de enseñanza de las Ciencias en las que los alumnos utilizan determinados procedimientos, ya sea en trabajos de campo o de laboratorio, al abordar estas actividades no se puede hablar de una metodología en concreto, sino que abarca una gama de posibilidades" (Puentes, 2008).

\footnotetext{
${ }^{5}$ Al respecto se recomienda revisar Autobiografía de Charles Darwin y "The Variation of Animals and Plants Under Domestication" (variaciones en plantas y animales domesticados)

${ }^{6}$ Asintótico: que intenta alcanzar un punto, y cada vez se acerca más a ese punto, sin nunca alcanzarlo. Para el caso en cuestión, la experimentación en Biología funcional intenta alcanzar en algunas ocasiones el estatus de experimento en el sentido "estricto" (aislar y manipular variables).
} 
Los trabajos prácticos suelen referirse a ciertas actividades en el ámbito escolar y extraescolar en la que los estudiantes utilizan ciertos procedimientos para resolver situaciones planteadas en el contexto de enseñanza y que muchas veces se relacionan con las salidas de campo o con el trabajo al interior del laboratorio. (Del Carmen, 2000).

Con respecto a los trabajos prácticos se encuentran diversas posiciones, como que pueden constituirse en actividades relevantes en la enseñanza por su pluralidad en cuanto a objetivos, como lo son la familiarización, observación e interpretación de diferentes fenómenos (Caamaño, 2003). También hay quienes consideran que los trabajos prácticos pueden ser poco relevantes y hasta una pérdida de tiempo en la enseñanza de las Ciencias (Hodson, 1994).

Con respecto a los diferentes argumentos expuestos en los párrafos de este apartado, el desarrollo de la tesis de este ensayo no pretende dilucidar las diferentes argumentaciones como si son o no son apropiados los trabajos prácticos en la enseñanza de la Biología, o si son los trabajos en el laboratorio o las salidas de campo los únicos tipos de trabajos prácticos, sino mas bien la intencionalidad de este ensayo es reconocer que existen los trabajos prácticos como una estrategia didáctica utilizada en el aula, y que en consecuencia para los docentes de Biología, sería recomendable reconocer y reflexionar sobre si a estos trabajos prácticos se les está dando un enfoque desde la Biología evolutiva o la Biología funcional, o una mezcla de ambas en pos de favorecer los procesos de enseñanza-aprendizaje de la Biología en la escuela.

Cuando se enseña Biología evolutiva a los estudiantes por medio de trabajos relacionados con salidas de campo, por ejemplo, los alumnos podrían llegar a dar mayor valor científico a los métodos naturalísticos como la observación y la comparación. En este contexto sería recomendable que el profesor relacionara muy adecuadamente los aspectos teóricos, epistemológicos e históricos de las metodologías comparativas y observacionales con los estudiantes con el fin de que los estudiantes no construyan un imaginario de Ciencia biológica inadecuado, como por ejemplo que los estudiantes relacionaran biólogo únicamente con "naturalista" (observador-contemplativo). Sería también recomendable que los docentes de Biología relacionaran los estudios realizados en las salidas de campo con posteriores análisis y experimentaciones en el laboratorio, para de esta manera no crear una brecha innecesaria entre la Biología evolutiva y la funcional.

De igual manera un docente que sólo le de valor al trabajo y procedimientos propios de la Biología funcional, como las experiencias de laboratorio, podría crear un imaginario de Biólogo distorsionado en los estudiantes.

Igualmente los docentes de Biología ya sea que se esté enseñando Biología evolutiva o funcional deberían intentar no dejar que los estudiantes le resten valor a las teorías, presupuestos o conjeturas que se deben tener antes de llegar a hacer las observaciones 
para no caer "en la trampa inductivista de considerar la observación como objetivo y punto de partida del método científico, punto de vista fuertemente refutado por la filosofía actual de la Ciencia" (Miguens y Garrett, 1991, p.231).

Se tendría que para unificar los procedimientos propios en prácticas orientadas desde la Biología evolutiva y la Biología funcional en pos de favorecer los procesos de enseñanzaaprendizaje de los estudiantes, dar el correspondiente valor a la observación y comparación científica y a los procedimientos propios de la experimentación, dentro de una adecuada construcción de diseños experimentales que puedan dar cuenta de hipótesis, resultados, generalizaciones, hasta donde sea posible, para que así se logre construcción de conocimiento significativo en los estudiantes. A este respecto Orange (1999) citado por Dourado (2006) habla de qué aspectos deben ser tenidos en cuenta para una correcta implementación de las actividades propias del laboratorio con los trabajos de campo, correspondiendo los primeros al enfoque funcional y los segundos al evolutivo, aclarando que no son los únicos aspectos metodológicos procedimentales de cada enfoque.

- Ambos trabajos prácticos, deben generar un contacto de los alumnos con las formas "reales" (de campo o laboratorio) que proporcione una utilización de estrategias de resolución de problemas reales o que provengan de lo real.

- Un trabajo de laboratorio debe orientar, naturalmente, una lectura de los datos de campo y, en contrapartida, una actividad de campo permitirá un análisis crítico de los resultados obtenidos en el laboratorio.

- Los datos obtenidos en el campo, y los datos obtenidos en el laboratorio, deben ser influenciados por referentes teóricos propios de cada enfoque.

- La solución de los problemas a estudiar, resultará en la articulación entre los datos empíricos recogidos en el campo, o en el laboratorio, y los modelos teóricos disponibles.

- Los trabajos de laboratorio, o de campo, contribuyen para la resolución de problemas de un modo desigual, debido a que el tiempo en el laboratorio o en el campo suele ser diferente.

Para terminar este ensayo, se retomará una hermosa argumentación realizada por Telleira, en su ensayo "Batas y botas. La cercana lejanía de las dos Biologías". Telleira utiliza este juego de palabras del título, para referirse a que los biólogos evolutivos son las "botas", debido a su constante trabajo de campo, y los biólogos funcionales son las "batas", por su constante trabajo en el laboratorio y que dice: 
"La biología funcional está dominada por la manipulación en condiciones de laboratorio (de ahí viene la bata...), donde pueden repetirse a voluntad y relativa rapidez los procesos en estudio. Pero como no se pueden replicar los procesos históricos que han configurado la biodiversidad actual, la biología evolutiva plantea sus hipótesis bajo la forma de narrativas histórica y usa el método comparado o la investigación de campo (de ahí viene la bota...) para testar sus predicciones." (Telleira, 2006)

Sin embargo:

"Debemos aspirar a un plan donde el nivel formativo de nuestros alumnos sea algo más que la suma de unas piezas impartidas desde nuestras particulares visiones la Biología. No propongo la falta de especialización ni mucho menos, sino una búsqueda activa de la necesaria conexión entre las diversas aproximaciones al estudio de la vida".(Telleira, 2006).

\section{BIBLIOGRAFÍA}

Caamaño, A. (2003). Los trabajos prácticas en ciencias. pp.95-105 En: Jiménez, P. (Compiladora). Enseñar Ciencias. Barcelona: Graó, 322 p.

Canguilhem, G. (1976). El conocimiento de la vida. Capítulo “La experimentación en Biología Animal". Barcelona. Anagrama. 421 p.

Caponi, G. (2003). Experimentos en Biología Evolutiva: ¿Qué tienen ellos que los otros no tengan? Episteme, Porto Alegre, 1(16): 61-97

Darwin, Charles R. (2007) [1881]. La Fecundación de las Orquídeas. Madrid: Laetoli. 446 p.

Darwin, Charles R. (1883). The Variation of Animals and Plants Under Domestication. Second Edition, Revised Charles Darwin New York: D. Appleton \& Co. 567 p.

Darwin, Charles R.(2007) [1929]. Autobiografía de Charles Darwin: Apéndices y adaptación por Sir Francis Darwin. Basada en las notas inéditas de Charles Darwin (1876). Bogotá: Norma. 158 p.

Del Carmen, L. (2000). Los trabajos prácticos. pp. 267-288. En: Perales, F., Cañal, P (Compiladores). Didáctica de las Ciencias Experimentales. Teoría y práctica de la Enseñanza de las Ciencias. Alcoy: Marfil. 432 p.

Díaz, J. (2002). El Método Comparativo en la Biología Evolutiva. Etologuia, Madrid, 19 (20): $1-46$. 
Dourado, Luis. (2006). Concepçoes e prácticas dos profesores de Cièncias Naturais relativas à implementaçao integrada do trabalho de campo. Revista Electrónica de Enseñanza de las Ciencias 1(5): 192-212

Hodson, D. (1994) Hacia un enfoque más crítico del trabajo de laboratorio. Enseñanza de las Ciencias. 12 (3):299-313.

Mayr, Ernst. (1995). Así es la biología. Madrid: Debate.326 p.

Mayr, Ernst. (2006). Por qué es única la biología. Consideraciones sobre la autonomía de una disciplina científica. Buenos Aires: Katz. 280 p.

Miguens, M. y Garrett, R. (1991).Prácticas en la Enseñanza de las Ciencias. Enseñanza de las Ciencias. 9 (3):229-236.

Orange, C. et al. (2001). Trabalho Laboratorial no Ensino da Fisica: avaliaçao da formaçao e do desempenho de futuros profesores. Tese de mestrado, Universidae do Minho.

Puentes, M. (2008). Propuesta de un sistema de categorías para el estudio de los trabajos prácticos en la Enseñanza de la Biología. Trabajo de Grado de Especialización en Enseñanza de la Biología. Universidad Pedagógica Nacional, Bogotá.

Telleira, J. (2006). Batas y botas. La cercana lejanía de las dos Biologías. En 50 promoción Facultad de Biología UCM. Visto 2 de diciembre de 2008 en: http://darwin.bio.ucm.es/usuarios/50promo/cont/memorias0021.htm 